\title{
EVALUACIÓN DE LA EFICIENCIA, EFICACIA Y EQUIDAD SOCIAL DEL IMPUESTO PREDIAL EN EL MUNICIPIO DE SOGAMOSO, CON BASE EN LA ÚLTIMA FORMACIÓN CATASTRAL
}

(Evaluation of efficiency, effectiveness and social equity of the property

tax in the municipality of Sogamoso, based on the last cadastral update)

Juan Sebastián Izaquita Orduz* Rigoberto Antonio Bernal Burgos**

\author{
*Contador Público, Escuela de Contaduría Pública, UPTC. Grupo GICONT, jenny.rosales@uptc.edu.co. \\ ** Magíster en Economía, docente Contaduría Publica UPTC Facultad seccional Sogamoso.
}

(Recibido: el 27 de Febrero de 2013 y aceptado 22 de Octubre de 2013)

\section{Resumen:}

El presente proyecto de investigación se fundamentó en la evaluación de los niveles de eficiencia, eficacia y equidad social del proceso de recaudo por concepto de impuesto predial en el municipio de Sogamoso, tomando como base la última formación catastral. Básicamente, se estableció el monto causado por concepto de este impuesto antes de la última formación catastral, y se comparó con el monto efectivamente recaudado en este mismo período, así como los recursos usados para lograr el recaudo de cada vigencia. El resultado de esta comparación determinó, en términos generales, que el nivel de eficacia es satisfactorio en cuanto al dinero, pero no lo fue en relación con los predios, y que la eficiencia del proceso de recaudo fue considerablemente alentadora.

En cuanto a los metros construidos desde la última formación catastral, se estableció su evolución entre cada actualización catastral. Además, se hizo un análisis al componente de equidad social que implica el recaudo del impuesto predial, lo que constituyó un elemento de suma importancia para determinar el grado de eficiencia y eficacia que se pretendía establecer. Además de mostrar la evolución del recaudo, este proyecto arrojó resultados que serán determinantes dentro de los planes de acción y ejecución presupuestal para la actual Administración en materia tributaria en general y de impuesto predial en particular.

Palabras clave: avalúo, eficacia, eficiencia, equidad social, intereses, descuentos, acuerdo municipal, impuesto predial, urbano, rural, suburbano, predios de expansión.

\section{Abstract:}

This research project was based on the assessment of the levels of efficiency, effectiveness and social equity of the tax collection process on property in the city of Sogamoso, based on the last cadastral update. Basically, it was established the amount caused by this tax before the last cadastral update and was compared with the actual amount in the same period, as well as the resources used to achieve the collection of each fiscal year. The result of this comparison broadly determined that the level of effectiveness is satisfactory in terms of money but it was not in terms of number of properties, and that the efficiency of the collection process was greatly encouraging.

In terms of meters built since last cadastral update, it was established its evolution between each cadastral update. In addition, it was analyzed the social equity component which involves the collection of property tax, and which was an important element in determining the degree of efficiency and effectiveness that was intended to establish. Besides showing the evolution of the collection, this project presents results that will be decisive in action and budget execution plans for the current tax administration in general and for property tax in particular.

\section{INTRODUCCIÓN}

No es solo la controversia que ha generado el cobro del impuesto predial unificado para la presente vigencia lo que motivó este estudio, fue también el constante rezago, la desinformación y la falta de interés de los que pareciera ser objeto este impuesto en Sogamoso, por el hecho de tener una periodicidad anual y un lapso de doce vigencias sin actualización catastral. La razón de ser de este documento es, del mismo modo, la necesidad de conocer y dar a conocer el resultado de un diagnóstico tributario de un tema poco abordado investigativamente desde la academia, que capta la atención de una comunidad después de catorce años, y la importancia de estar al tanto respecto de un tributo tan inclusivo como el predial.
En este sentido, el análisis que se desarrolló, seguramente se convertirá en una herramienta influyente para la toma de decisiones al respecto, en tiempo presente y futuro, pese a estar fundamentado en hechos del pasado. Saber si el proceso de recaudo del impuesto predial se lleva a cabo como debiera, más allá de servir como un reporte con destino a la Administración, pretende llegar a ser una carta de navegación municipal en materia tributaria, financiera y de gestión, un espaldarazo al crecimiento y desarrollo de un territorio que tiene solo un representante, pero miles de responsables. 


\section{IMPUESTO PREDIAL}

\subsection{Aspectos preliminares}

Como es bien sabido, la base para el cálculo del impuesto predial en Colombia es el avalúo que arroja la formación catastral. Esta consiste en la aplicación de un estudio mediante el cual un predio, ya sea lote, terreno y construcciones tales como casa, apartamento, bodega o cerramiento, es visitado por los peritos del Instituto Geográfico Agustín Codazzi, Sección Catastral, para ser registrado con la información física, jurídica y económica del bien.

El avalúo consiste en la determinación del valor de los predios. Para la determinación del valor del terreno, se tienen en cuenta variables como la norma de uso del suelo, la actividad económica del sector, las vías y su estado, los servicios públicos disponibles y la topografía, entre otros. Para las construcciones se consideran el tipo de estructura, los acabados, el baño, la cocina, el estado de conservación, la edad de la edificación y el uso que se le da, lo cual es valorado de acuerdo con las condiciones del inmueble. Según el artículo $5^{\circ}$ de la Ley 14 de 1983 y el artículo 24 de la Ley 1450 de 2011, formar o actualizar los catastros es una labor que deben cumplir los municipios colombianos cada cinco años. Sin embargo, en el caso de Sogamoso, las últimas formaciones catastrales se llevaron a cabo en los años 2000 y 2013.

Uno de los aspectos más ambiguos del impuesto predial es la variación entre el avalúo de los predios, o, dicho de otro modo, por qué un predio vale más que otro. La razón está en que todas las propiedades no son iguales, sus dueños realizan cambios, adiciones y mejoras a sus viviendas. Además, unos barrios se transforman más aceleradamente que otros en materia de infraestructura y los cambios en el uso del suelo, sin dejar de lado un factor determinante como el la diferenciación en las condiciones del mercado inmobiliario en los diferentes sectores de la ciudad. Sin embargo, según el artículo 6 de la Ley 44 de 1990, "A partir del año en el cual entre en aplicación la formación catastral de los predios, en los términos de la Ley 14 de 1983, el Impuesto Predial Unificado resultante con base en el nuevo avalúo, no podrá exceder del doble del monto liquidado por el mismo concepto en el año inmediatamente anterior, o del impuesto predial, según el caso", lo que indica que el impuesto predial no solamente tiene un rango en pesos por mil sino también un tope en pesos reales.

El otro punto álgido de la liquidación de este tributo es la estructura tarifaria de cada municipio. Esta estructura es determinada por el concejo municipal y varía de manera diferencial y progresiva, fundamentada en criterios como el estrato socioeconómico, usos del suelo, antigüedad de la actualización, rango de área y el avalúo catastral (Ley 1450 de 2011, art. 23).

Sin embargo, la ley no contempla expresamente las condiciones de ingresos de los contribuyentes propietarios de finca raíz, y eso se manifiesta en algún grado de inequidad con relación a personas que son económicamente inactivas o cuya capacidad de pago es insuficiente.

En Sogamoso se pretenden analizar los acuerdos municipales de impuesto predial, y con base en las condiciones socioeconómicas de los propietarios, conocer el grado de equidad social de este tributo.
El documento que rige los aspectos de impuesto predial unificado en el municipio de Sogamoso, es el Acuerdo Municipal 065 de 2005, por medio del cual se adoptó el Estatuto Tributario, se compilaron las normas que conforman el Estatuto de Rentas del Municipio.

A la fecha, en materia de impuesto predial, este documento ha experimentado dos modificaciones. La primera, con el Acuerdo 071 de 2006, que cambió tres de sus artículos; la segunda, con el Acuerdo 024 de 2012, cuyos primeros siete artículos realizan modificaciones, entre ellas a componentes tan importantes como la estructura tarifaria, adicionan incentivos, exclusiones, exenciones, e incluso, derogan dos de los artículos del Acuerdo 065 de 2005.

La investigación comenzó con una comparación entre el monto causado por concepto de impuesto predial (de los períodos precedentes a la última formación catastral), con respecto al monto efectivamente recaudado, de tal suerte que, este diagnóstico permitió establecer el nivel de eficiencia en el proceso de recaudo del período anterior, $\mathrm{y}$, a partir de ello, se procedió a diagnosticar el período posterior a la última formación catastral en el municipio de Sogamoso. Esto con el fin de servir de apoyo a la Administración Municipal en la toma de futuras decisiones en materia de impuesto predial unificado como herramienta para la gestión tributaria fiable y objetiva.

\subsection{Diagnóstico del recaudo}

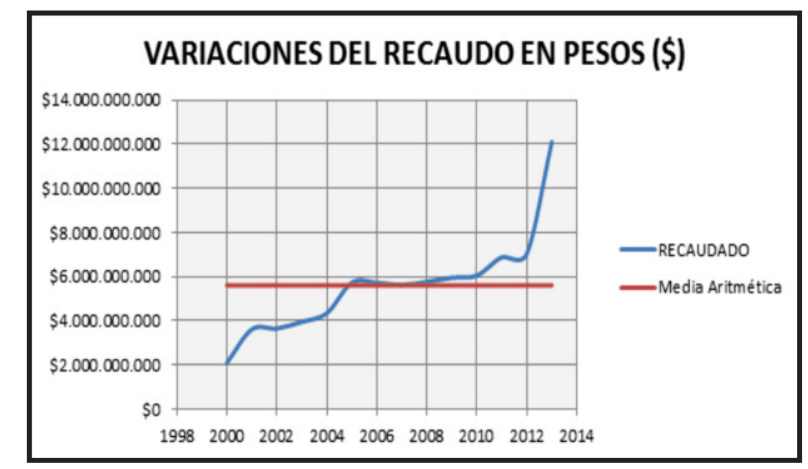

Figura 1. Variaciones del recaudo: años 2000- 2013.

Fuente: Secretaría de Hacienda de Sogamoso, Oficina de Sistemas (2013).

En la Figura 1 se observa el comportamiento del recaudo por impuesto predial del 2000 al 2013 en el municipio de Sogamoso. La Administración Municipal pasó de recaudar siete mil treinta millones cuatrocientos sesenta y seis mil pesos corrientes $\left(\$ 7.030^{\prime} 466.000\right)$ a doce mil noventa y dos millones cuatrocientos dos mil pesos corrientes ( $\left.\$ 12.092^{\prime} 402.000\right)$, lo cual representa un incremento de cinco mil sesenta y un millones novecientos treinta y seis mil pesos corrientes (\$5.061'936.000), el más significativo de los últimos trece años de recaudo por impuesto predial unificado en Sogamoso. Este esfuerzo fiscal predial, se debe principalmente a la actualización catastral aplicada en el año 2013, que acrecentó significativamente los avalúos de los predios del municipio, $\mathrm{y}$, en consecuencia, el valor del impuesto a pagar $\mathrm{y}$, por ende, el incremento del recaudo. Vale la pena aclarar que la cifra del recaudo del año 2013 es parcial, toda vez que corresponde al monto registrado a 31 de mayo de 2013 y, desde luego, no tiene en cuenta las contribuciones que se hagan en el resto de la vigencia. Así mismo, es menester mencionar que el proceso de actualización catastral no se llevaba a cabo en Sogamoso desde el año 2000, pese a que el artículo $5^{\circ}$ de la Ley 14 de 1983 determina que tal reajuste debe realizarse cada cinco años.
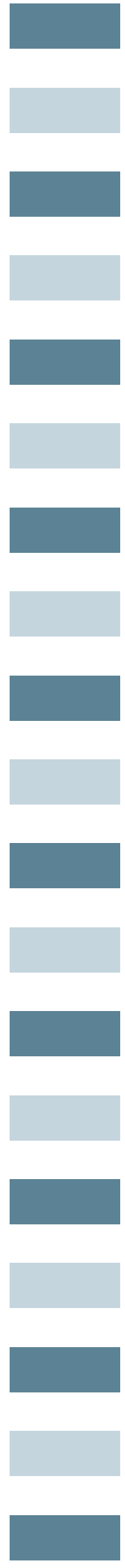
La Figura 4 muestra la relación costo-beneficio del recaudo con respecto al valor de la nómina de la Secretaría de Hacienda y Gestión Financiera. El procedimiento que se llevó a cabo para determinar el costo específico para el recaudo del impuesto predial unificado, fue el siguiente:

Inicialmente, se determinó el porcentaje de participación del impuesto predial en el total de los ingresos tributarios de cada vigencia. Ese porcentaje se le aplicó al valor de la nómina del Departamento de Hacienda y Gestión Financiera, lo que técnicamente da como resultado la suma de dinero cancelada a este personal por recaudar concretamente el impuesto predial. Ahora bien, la gráfica demuestra un comportamiento positivo en este sentido, dado que las vigencias cuyos recaudos resultaron más costosos fueron las de 2002 y 2004, con apenas cuatro centavos por cada peso recaudado. Las demás vigencias maniobran un costo que fluctúa entre los dos y tres centavos por peso recibido.

En ese orden de ideas, una actividad o un proceso no son eficientes cuando usan recursos estimados en un valor igual o superior al del objetivo alcanzado, lo que quiere decir que, si partimos del hecho de que alcanzar el $100 \%$ de eficiencia consiste en alcanzar el objetivo propuesto haciendo el mínimo uso de los recursos disponibles, se concluye que a través de los últimos catorce años la Administración Municipal ha recaudado el impuesto predial unificado con una eficiencia del $97,75 \%$.

La conducta de la línea que representa el valor que la Administración Municipal ha tenido que pagar a su personal por recaudar el impuesto predial de cada propiedad (ver Figura 5), ha sido notablemente alterada, sobre todo hasta el año 2007. Entre la vigencia 2001 y la de 2002 se presentó un incremento destemplado, ya que la Administración pasó de pagar \$2.424 por recaudar el impuesto de un predio, a pagar $\$ 4.073$ por el mismo concepto. El pico más alto de la gráfica en cuestión corresponde al año 2004, en el que cada predio que canceló su obligación físcal le costó al municipio $\$ 5.014$; desde entonces, no se presentaron aumentos significativos en este sentido. Desde la vigencia 2007, el costo se mantuvo entre los $\$ 3.004$ y $\operatorname{los} \$ 3.366$.

El valor que ha pagado, en promedio, cada predio año a año (ver Figura 6) constituye la contracara para la evaluación de la eficiencia en los mismos términos.

Entre el año 2000 y 2001 se presentó el cambio más importante en el recorrido de la línea, que equivale a un incremento de $\$ 47.851$. El punto más alto se contempla en la vigencia 2012 en la que, con corte a 31 de diciembre, cada predio había pagado $\$ 178.738$ por impuesto predial.

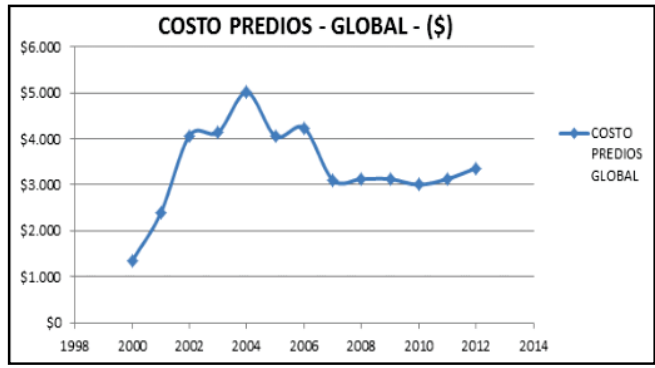

Figura 5. Costo promedio (pesos corrientes)

Fuente: Secretaría de Hacienda de Sogamoso, Oficina de Sistemas (2013).

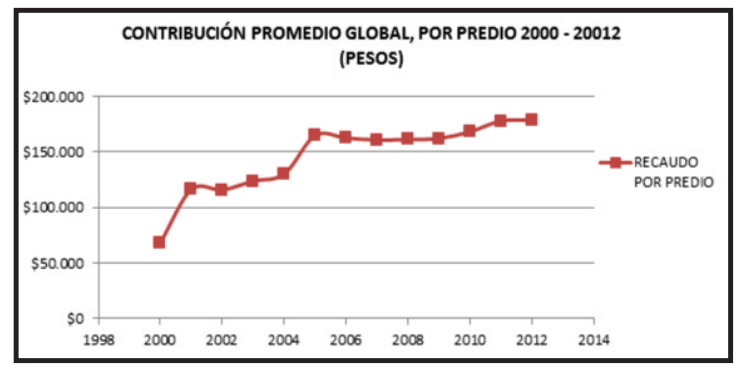

Figura 6. Contribución promedio (pesos corrientes).

Fuente: Secretaría de Hacienda de Sogamoso, Oficina de Sistemas (2013).

\subsection{Equidad social}

En términos generales, se podría asumir que la equidad social merece el mismo significado que la igualdad social. Esta última es una situación social en la cual las personas tienen las mismas oportunidades o derechos de acceso al disfrute del bienestar general. No obstante, en el campo tributario, la diferencia es considerablemente sustancial, toda vez que la igualdad supone una tributación de igual cuantía para todos los contribuyentes, el llamado impuesto Lump Sum. Un claro ejemplo de igualdad tributaria es la tarifa del IVA, porque cada uno, sin importar su nivel de ingresos, su nivel de vida, debe pagar el $16 \%$ o la tarifa que corresponda al bien o servicio adquirido. Mientras que la equidad es un criterio que persigue una carga selectiva acorde con la capacidad de pago de cada contribuyente, como es el caso del impuesto de renta para personas naturales. La equidad, en términos tributarios, implica una desigualdad; esa es la paradoja semántica que se percibe entre estos dos conceptos.

En la Figura 2 se observa que las metas en pesos corrientes son superadas en siete de las catorce vigencias objeto de estudio. Sin embargo, este comportamiento no es nada compatible con el recaudo expresado en términos de contribuyentes (Figura 3), que no tuvo ni una sola vigencia eficaz, y que durante los años sometidos a evaluación sumó un total de 258.125 predios que dejaron de pagar el impuesto predial, para una eficacia agregada del $63,54 \%$.

Esto supone una diferencia con respecto al rendimiento en pesos corrientes (\$) del 41,96\%, y visto desde la óptica de la equidad social, significa que durante este período, 258.125 contribuyentes se han beneficiado de los recursos que han tributado los demás; que el hecho de recaudar más dinero del esperado no debe ser para la Administración una garantía de eficacia ni mucho menos de equidad; que esa misma equidad no es una virtud exclusiva de las alcaldías, sino que también debe formar parte de la cultura tributaria de los contribuyentes y que frente a su compromiso fiscal con el municipio, la finca raíz sogamoseña está cada vez más lejos de ser considerada morosa y cada vez más cerca del escandaloso rótulo de la evasión.

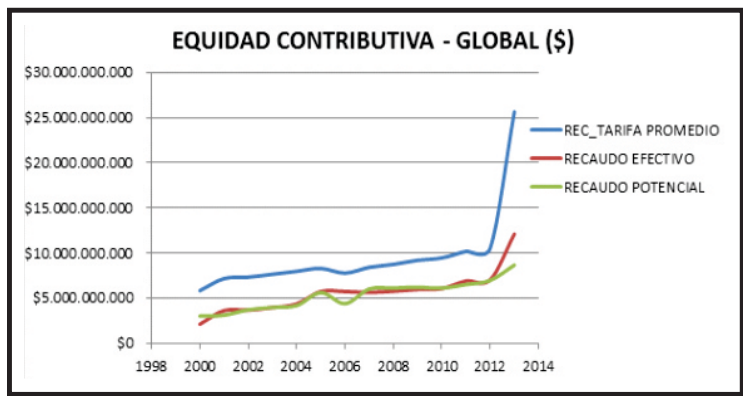

Figura 7. Equidad contributiva respecto a tarifa promedio (pesos corrientes). Fuente: Secretaría de Hacienda de Sogamoso, Oficina de Sistemas (2013). 
Según la Figura 7, los sogamoseños situaron su recaudo muy por debajo de la línea correspondiente a la tarifa promedio. Esta última corresponde a los valores que se hubieran recaudado si, en promedio, todos los predios formados pagaran oportunamente la misma tarifa de acuerdo con la estructura tarifaria vigente para cada año.

Como se ve, los montos proyectados para cada año por la Administración son inferiores al recaudo por tarifa promedio; la diferencia entre uno y otro a través de los catorce años evaluados es de \$59.653'067.000, lo que demuestra que las metas impuestas por el fisco municipal en materia de impuesto predial podrían ser más ambiciosas.

Pese a que el monto efectivamente recaudado se acerca más al recaudo sugerido por tarifa promedio: \$55.563'346.000 por debajo de éste, queda claro una vez más que si bien es cierto que el recaudo es generalmente tranquilizante en dinero, no lo es en número de contribuyentes; dicho de otro modo: pagan todo, pero no todos.

\section{CONCLUSIONES}

1. El notable nivel de eficacia alcanzado por la Administración durante los últimos catorce años, se debe en gran medida a que esta se propone metas demasiado bajas para cada vigencia. Para el año 2013, el valor a recaudar se incrementó en menor proporción que el valor efectivamente recaudado, de tal suerte que esta vigencia contribuye en gran medida a que, en suma, Sogamoso haya logrado una vistosa eficacia del 105,50 $\%$.

2. Una estructura tarifaria basada en rangos de avalúo se encaja mejor en el contexto de equidad social, que una estructura basada en zonas o grupos de sectores.

3. La equidad social, entendida como una cualidad del sistema tributario municipal, no debe atribuírsele exclusivamente al sujeto activo, sino también al sujeto pasivo, como manifestación de cultura tributaria para con el fisco de su municipio y para con su comunidad.

4. Los tres conceptos alrededor de los cuales giró este estudio: eficacia, eficiencia y equidad social, se relacionan entre sí de una manera natural, no solo en el plano tributario sino en todas las actividades de la cotidianidad.

5. El contador público tiene todavía muchos potenciales objetivos de investigación en el sector público y debe poner sus capacidades al servicio y en función de una comunidad de la que él también es parte.

\section{RECOMENDACIONES}

1. Establecer los recaudos potenciales teniendo en cuenta el saldo que se dejó de recaudar en la vigencia anterior, de modo que tales objetivos vayan en alza y no se presenten comportamientos como el del año 2006 (ver Figura 7), en el cual el valor que se esperaba recaudar, disminuyó. En efecto, este comportamiento afecta la noción objetiva de la efícacia.

2. Hacer lo posible para segmentar los recaudos por estratos socioeconómicos, para facilitar futuros estudios sobre la equidad social y para contribuir a la toma de decisiones en materia de impuesto predial, o para soportar las decisiones tomadas al respecto.
3. Realizar charlas informativas para que la comunidad sogamoseña conozca el funcionamiento del impuesto predial, las entidades que intervienen para hacer posible su recaudación, los derechos que tienen como contribuyentes y el compromiso constante del que son parte.

4. Cada Administración debe hacer cuanto sea posible para que el proceso de actualización catastral no se postergue por más de cinco años, como lo establece la Ley 14 de 1983, esto con el fin de evitar cambios tan abruptos en los avalúos como los presentados para el año 2013, que han causado malestar social en los contribuyentes por el alza exagerada del tributo aunque también actualización al valor real de sus predios.

\section{REFERENCIAS}

Concejo Municipal de Sogamoso. (1999). Acuerdo 086. Artículo 24.

Concejo Municipal de Sogamoso. (1999). Acuerdo 087.

Concejo Municipal de Sogamoso. (2000). Acuerdo 005.

Concejo Municipal de Sogamoso. (2001). Acuerdo 050.

Concejo Municipal de Sogamoso. (2001). Acuerdo 078.

Concejo Municipal de Sogamoso. (2001). Acuerdo 080.

Concejo Municipal de Sogamoso. (2001). Acuerdo 013.

Concejo Municipal de Sogamoso. (2005). Acuerdo 065. Artículos 1 al 44.

Concejo Municipal de Sogamoso. (2006). Acuerdo 071. Artículos 1 al 3.

Concejo Municipal de Sogamoso. (2012). Acuerdo 024. Artículos 1 al 7.

Congreso de la República. (1983). Ley 14. Artículos 1 al 31.

Congreso de la República. (1986). Ley 75. Artículos 73 al 77.

Congreso de la República. (1990). Ley 44. Artículos 1 al 9.

Congreso de la República. (1995). Ley 24. Artículo 6.

Congreso de la República. (1997). Ley 388. Artículos 1 al 139.

Congreso de la República. (2011). Ley 1450. Artículos 23 y 24.

Corte Constitucional de Colombia. (2001). Sentencia C-1060A

Corte Constitucional de Colombia. (2002). Sentencia C-734/02.

Corte Constitucional de Colombia. (2002). Sentencia C-643/02.

Constitución Política de Colombia. (1991). Artículo 363.

Iregui, A. M., Melo, L., Ramos, J. (2003). El impuesto predial en Colombia: evolución reciente, comportamiento de las tarifas y potencial de recaudo. Bogotá: Banco de la República. 